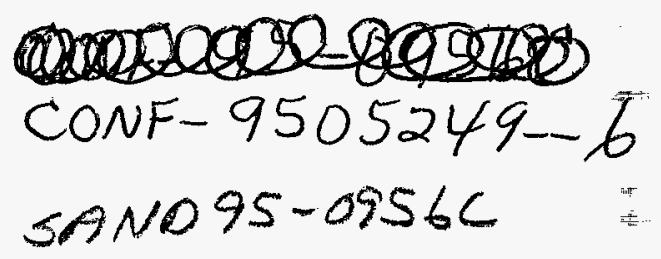

\title{
NUMERICAL SIMULATION OF GRAIN SIZE DISTRIBUTIONS IN LIQUID PHASE SINTERED MATERIALS
}

\author{
Veena Tikare \\ Sandia National Labs., Albuquerque, NM 87185-1411 \\ and \\ James D. Cawley \\ Case Western Reserve Univ., Cleveland, OH 44106
}

\begin{abstract}
Grain growth in liquid phase sintered materials by Ostwald ripening has been modeled extensively by both analytical and numerical techniques. However, all models make simplifying approximations to make the problem tractable and the approximations used in these models make them most accurate at very low solid fractions. A two-dimensional, Monte Carlo simulation technique based on the Potts model that makes no assumptions about solid fraction, grain shapes or diffusion fields around grains has been used to study grain growth in fully wetting, liquid phase sintered systems. The grain size distribution, GSD, was found to vary with solid fraction, becoming broader and more peaked with increasing solid fraction. The skewness was near zero at solid fraction of 0.41 and shifted to larger grain sizes with increasing solid fraction.
\end{abstract}

\section{INTRODUCTION}

Many technologically important ceramics such as silicon nitride ceramics, alumina substrates and barium titanate electrical capacitors are liquid phase sintered. It is important to understand evolution of microstructural features generated by processes such as grain growth so that these materials may be engineered for their respective applications. Greenwood ${ }^{1}$ was the first to consider grain growth by Ostwald ripening, but the approach of Lifshitz \& Slyozov ${ }^{2}$ and of Wagner ${ }^{3}$ (LSW theory) is the basis of modern studies of this subject. In order to make the model tractable, the LSW theory assumes the distance between grains is much larger than the grain size (or equivalently the fraction of grains is zero) and a mean solute concentration in the liquid matrix. The LSW theory predicts a power law behavior for grain growth given by the following: 


$$
R^{n}-R_{o}^{n}=k t
$$

eq. 1

where $t$ is time, $R$ is grain radius at time $t, R_{0}$ is the grain radius at $t=0, k$ is a constant and, the grain growth exponent $\mathbf{n}=3$ for diffusion controlled grain growth. In addition, LSW predicts all systems will evolve to a unique steady state grain size distribution, GSD. Many investigators $s^{4}, 5,6,7$ have modified the LSW theory to include finite volume fraction of grains using a variety of techniques; however, they all make simplifying assumptions such as spherical grains at all solid fractions ${ }^{5-8}$, uniformly spaced grains ${ }^{5,6}$, mean field concentration ${ }^{5,6,8}$, and all grains of a given size experience the same local environment ${ }^{5,6}$. All have found the power law behavior given is eq. (1) to hold with the same exponent, $n=3$, as well as broadening and increasing skewness of the GSD with increasing solid fraction. However, the GSD predicted by each of these models varies. We attribute these variations to the various assumptions made by each. In this paper, a simulation technique is presented which makes no assumptions about the solid fraction, concentration gradients in the liquid matrix, grain shape or grain spacing. The GSD obtained from this technique are presented and compared to those predicted by other grain growth theories.

\section{SIMULATION METHOD}

The Q-state, Potts, Monte Carlo simulation technique adapted by Srolovitz et al. ${ }^{8}$ to study grain growth was modified to simulate grain growth in liquid phase sintered materials. The microstructure was represented digitally on a twodimensional, square lattice with periodic boundary conditions in both the $\mathrm{X}$ - and $Y$ - directions. Two phases, liquid matrix and solid grains, were simulated by populating the lattice with a two-component canonical ensemble with the two components designated as $\mathrm{A}$ and $\mathrm{B}$. Component $\mathrm{A}$, the primary component in the solid phase, was allowed to assume one of 100 degenerate states; while component $\mathrm{B}$, the primary component in the liquid, was allowed to assume only one state. These states may be considered crystallographic orientation for the purposes of these simulations. The Hamiltonian, $\mathrm{H}$, for the simulation was the sum of the interaction energy, $J$, between each site, $i$, and each of its eight first and second nearest neighbors, $\mathrm{j}$, as given by

$$
H=\frac{1}{2} \sum_{j=1}^{8} \sum_{i=1}^{N} J\left(q_{i}, q_{j}\right)
$$

where $q_{i}$ is the orientation of site $i$ and $N$ is the total number of sites. The interaction energies used in these simulations were $\mathrm{J}(\mathrm{B}, \mathrm{B})=0, \mathrm{~J}(\mathrm{~A}, \mathrm{~B})=1.0, \mathrm{~J}\left(\mathrm{~A}_{\mathbf{i}}\right.$, 


\section{DISCLAIMER}

Portions of this document may be illegible in electronic image products. Images are produced from the best available original document. 
$\left.A_{i}\right)=0$ and $J\left(A_{i}, A_{j}\right)=2.5$. These interaction energies where chosen to simulate a fully wetting system. The simulation steps were (1) choose a site at random; (2) choose a neighboring site at random; (3) if one is an A-site and the other a B-site, choose a new orientation for the A-site at random; if not, goto step 6; (4) consider the two sites exchanging places with each other; (5) use the Metropolis algorithm to determine the exchange probability, $\mathrm{P}$;

$$
\begin{array}{ll}
P=\exp -\frac{\Delta H}{k_{B} T} & \Delta H>0 \\
P=1 & \Delta H \leq 0
\end{array}
$$

where $\Delta H$ is the change in energy given by eq. (2), $\mathrm{kB}$ is Boltzmann's constant and $T$ is absolute temperature; (6) increment the number of steps by $1 / \mathrm{N}$. This simulation method resulted in material transport from one grain to another by solution at the receding grain, diffusion through the liquid matrix, and precipitation onto the growing grain. No solid state diffusion occurred.

The simulations were run at temperature, $\mathrm{kBT}=1.3$. The solid fraction, $\varnothing$, was varied from $\varnothing=0.41$ to 0.89 by changing the mole fraction of components A and B. Multiple simulation of size 300 X 300 sites were run at each solid fraction to generate a large number of grains.

\section{RESULTS}

Analysis of grain size as a function of time revealed the power law behavior for grain growth is obtained in these simulations with a grain growth exponent of $n=$ 3 , as predicted for diffusion-controlled grain growth. This exponent was found to be constant with solid fraction.

The GSD obtained from the simulations are plotted as normalized frequency versus normalized grain size, $\mathrm{R} /<\mathrm{R}>$, so that the GSDs may be directly compared. Dynamic scaling of the GSD was obtained for all solid fractions studied. The GSDs after 100,000 MCS are used for comparison in figure 1. The GSD varied with the solid fraction. It became broader and more peaked with increasing solid fraction. The GSD was skewed to the right at solid fraction, $\varnothing=$ 0.89 , and the direction of skewness shifted to the left with decreasing solid fraction.

\section{DISCUSSION}

The simulation results were in agreement with grain growth theories on the trend in the spread of grain sizes. As the solid fraction increased, the GSD 

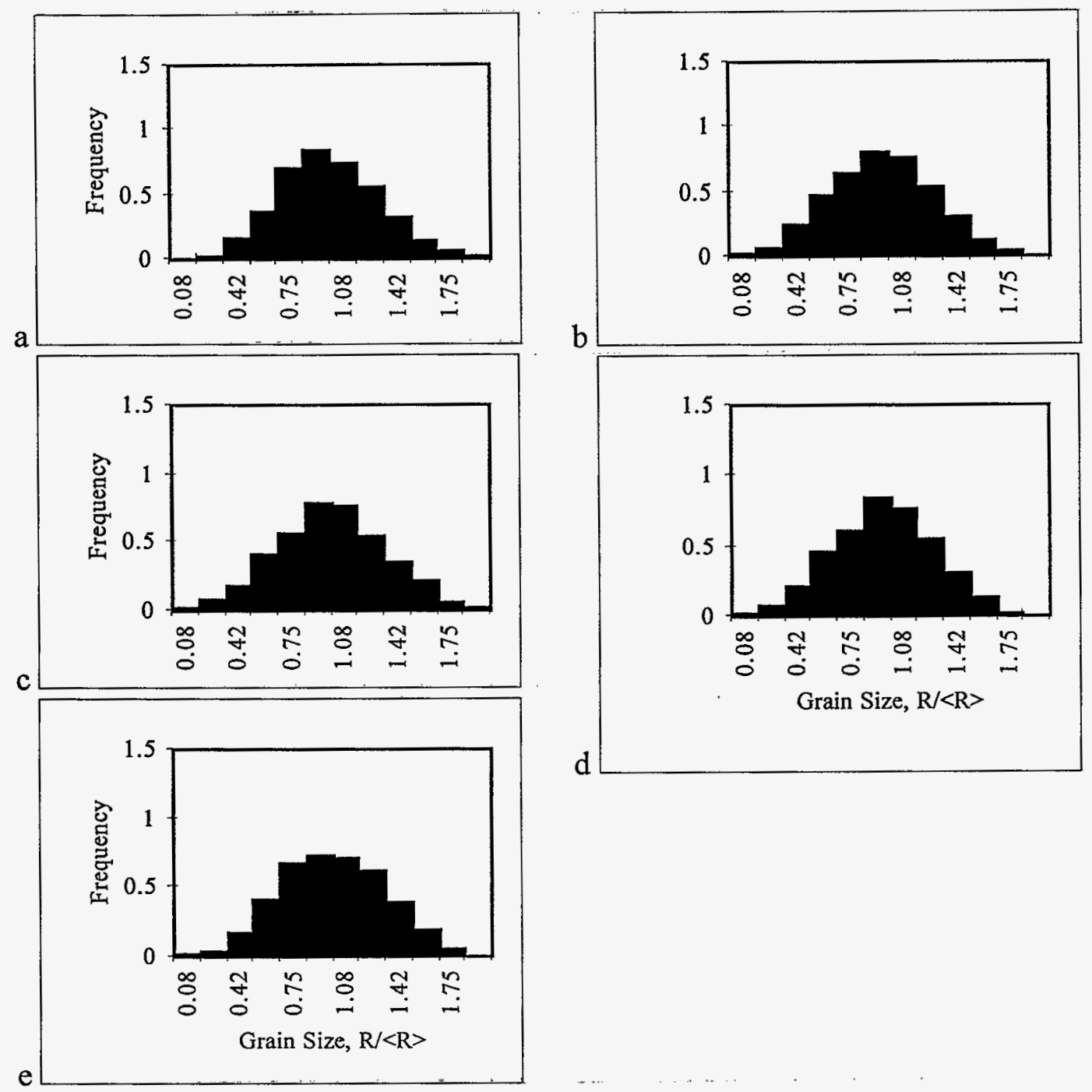

b

Figure 1. GSD's obtained from simulations at solid fractions of (a) 0.89 , (b) 0.78 , (c) 0.66 , (d) 0.53 and (e) 0.41 .

broadened because a few grains grew larger. The size of the largest grains increased from $1.9<\mathrm{R}>$ at $\emptyset=0.41$ to $2.2<\mathrm{R}>$ at $\varnothing=0.89$. This spread was larger than GSD's predicted by all grain growth theories except for Voorhees and Glicksman's 6

The skewness of the GSD obtained from simulation was calculated to be almost zero at a solid fraction, $\varnothing=0.41$ and increased with solid fraction to 0.3 at $\varnothing=0.89$. This is very different from the predictions of grain growth theories. At 
very low solid fraction, the LSW theory predicts a GSD with a negative skewness. The theories which consider finite solid fractions predict an increase in skewness, but not a change from negative to positive skewness as observed in the simulations. Figure 2a compares the GSD from simulations at solid fraction $\varnothing=$ 0.53 to that predicted by Voorhees and Glicksman. While, the spread in grain sizes were in agreement, the skewness was quiet different. The GSD obtained from simulations was skewed more to the larger sizes. The simulation GSD at $\varnothing$ $=0.66$ is compared to experimental data collected by Fang and Patterson ${ }^{9}$ for the $\mathrm{W}-14 \mathrm{Ni}-6 \mathrm{Fe}$ system at $\varnothing=0.60$ in figure $2 \mathrm{~b}$. They allowed the GSD to reach the steady state GSD and measured grain size by image analysis of two-dimensional cross-sections. The simulation GSD was in good agreement with the experimental data.

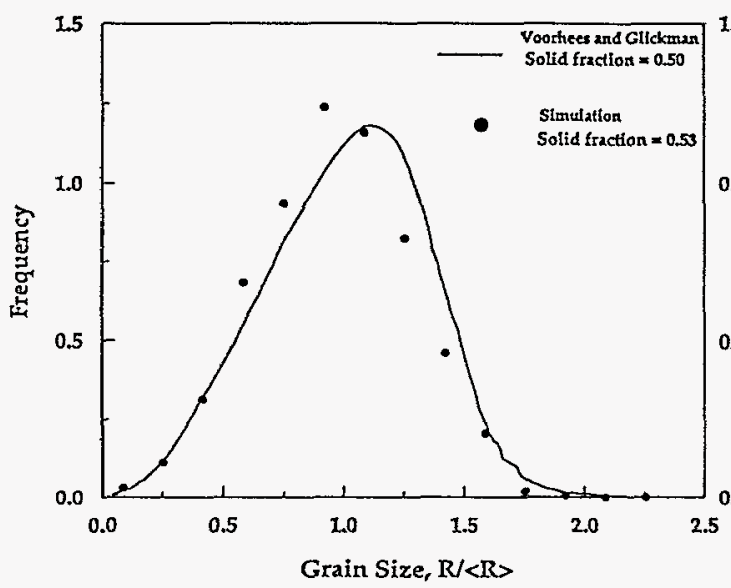

(a)

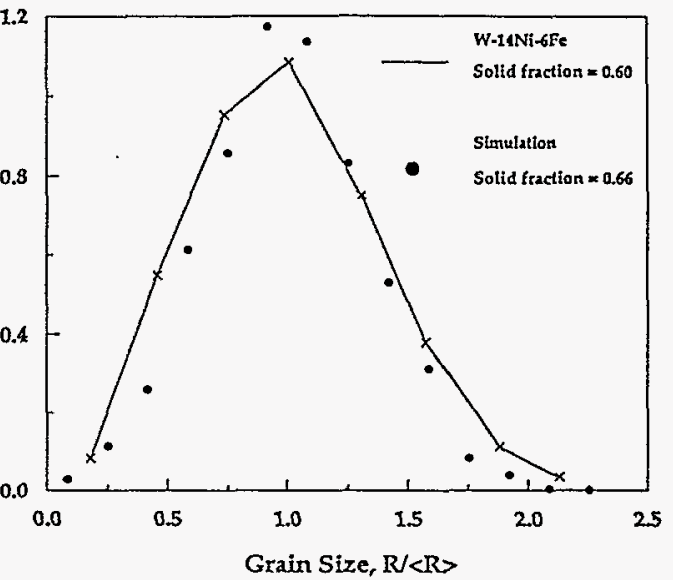

(b)

Figure 2. Comparison of GSD obtained from simulations to that obtained (a) by Voorhees and Glicksman and (b) experimentally by Fang and Patterson.

The advantage of the simulation technique presented in this investigation over that of other models was the spatial distribution of grains was allowed to develop naturally. Further, grain shape accommodation and solute gradients between grains were inherent to the technique. Voorhees \& Glicksman ${ }^{6}$ and Yao et al. ${ }^{7}$ considered spatial arrangement of grains, but they only considered diffusion gradients around spherical grains, at relatively low grain fractions where interactions were minimal. DeHoff's ${ }^{10}$ communicating neighbor model is, in principle, able to consider both deviation in curvature of grains and interactions of gradients between grains, but correlating curvature parameters and interaction parameters to nonspherical grain shapes has not been done. The ability of the simulation to incorporate spatial features may be the reason it was able to predict 
the change in skewness of the GSD from negative to positive with increasing solid fraction.

Another advantage of this simulation technique is that it has the ability to incorporate further microstructural complexities such as grain shape anisotropy and more than two phases. Such work is underway.

\section{CONCLUSIONS}

Grain Size distributions were characterized over a range of solid fractions, 0.40 to 0.90 . Under isotropic, fully wetting conditions, the grain size distribution varied with the solid fraction. It became broader and more peaked with increasing solid fraction. The grain size distribution was skewed to larger sizes at solid fraction $=0.89$. As the solid fraction decreased, the skewness shifted toward smaller sized grains.

\section{ACKNOWLEDGEMENT}

This work was partially performed at Sandia National Labs. and supported by the U.S. Dept. of Energy under contract number DE-AC04-94AL85000.

\section{REFERENCES}

${ }^{1}$ G.W. Greenwood, "The Growth of Dispersed Precipitates in Solution," Acta Metall., 4 243-248 (1956).

${ }^{2}$ I.M. Lifshitz and V.V. Slyozov, "The Kinetics of Precipitation From Supersaturated Solid Solutions," J. Phys. Chem. Solids, 19 [1-2] 35-50 (1961).

${ }^{3} \mathrm{C}$. Wagner, "Theory of the Aging of Precipitates by Redissolution (Ostwald Ripening)," Zeitschrift fur Elektrochemie, 65 [7-8] 581-591 (1961).

${ }^{4}$ A.J. Ardell, "The Effect of Volume Fraction on Particle Coarsening: Theoretical Considerations," Acta Metall., 20 61-71 (1972).

${ }^{5}$ A.D. Brailsford and P. Wynblatt, "Dependence of Ostwald Ripening Kinetics on Particle Volume Fraction," Acta Metall., 27 489-497 (1979).

"P.W. Voorhees and M.E. Glicksman, "Solution to the Multi-Particle Diffusion Problem with Application to Ostwald Ripening - II. Computer Simulations," Acta Metall., 32 [11] 2013-2030 (1984).

${ }^{7}$ J.H. Yao, K.R. Elder, H. Guo, and M. Grant, "Theory and Simulation of Ostwald Ripening," Phys. Rev., B 47 [21] 14110-14125 (1993).

${ }^{8}$ D.J. Srolovitz, M.P. Anderson, G.S. Grest and P.S. Sahni, "Grain Growth in Two Dimensions," Scripta Metall., 17 241-246 (1983).

${ }^{9}$ Z. Fang and B.R. Patterson, "Experimental Investigation of Particle Size Distribution Influence on Diffusion Controlled Coarsening," Acta Metall., 41 [7] 2017-2024 (1993).

${ }^{10}$ R.T. DeHoff, "A Geometrically General Theory of Diffuson Controlled Coarsening," Acta Metall., 39 [10] 2349-2360 (1991).

\section{DISCLAIMER}

This report was prepared as an account of work sponsored by an agency of the United States Government. Neither the United States Government nor any agency thereof, nor any of their employees, makes any warranty, express or implied, or assumes any legal liability or responsibility for the accuracy, completeness, or usefulness of any information, apparatus, product, or process disclosed, or represents that its use would not infringe privately owned rights. Reference herein to any specific commercial product, process, or service by trade name, trademark, manufacturer, or otherwise does not necessarily constitute or imply its endorsement, recommendation, or favoring by the United States Government or any agency thereof. The views and opinions of authors expressed herein do not necessarily state or reflect those of the United States Government or any agency thereof. 